\title{
The MURAVES project and other parallel activities on muon ab- sorption radiography
}

\author{
L.Bonechi1 $^{1,2, a}$, F.Ambrosino ${ }^{3,4}$, L.Cimmino ${ }^{3}$, R.D'Alessandro 2,1, G.Macedonio ${ }^{5}$, B.Melon ${ }^{1}$, \\ N.Mori $^{1,2}$, P.Noli ${ }^{3}$, G.Saracino ${ }^{4,3}$, P.Strolin ${ }^{4,3}$, F.Giudicepietro ${ }^{5}$, M.Martini ${ }^{5}$, M.Orazi ${ }^{5}$, and \\ R.Peluso ${ }^{5}$ \\ ${ }^{1}$ INFN Unit of Florence, Italy \\ ${ }^{2}$ Department of Physics and Astronomy - University of Florence, Italy \\ ${ }^{3}$ INFN Unit of Naples, Italy \\ ${ }^{4}$ Department of Physics and Astronomy - University of Naples "Federico II", Italy \\ ${ }^{5}$ INGV Osservatorio Vesuviano, Naples, Italy
}

\begin{abstract}
The MURAVES (MUon RAdiography of VESuvius) project is a joint activity participated by INGV, INFN and the Universities of Naples "Federico II" and Florence. The collaboration, following the experience gained within the previous INFN $\mathrm{R} \& \mathrm{D}$ project Mu-Ray, is currently completing the production of a robust four square meter low power consumption detector to be installed on the flank of Mount Vesuvius, an active volcano located on the western coast of Italy. The detector is supposed to collect data for at least one year, thus allowing performing a scan of the structure of the Vesuvius volcanic cone. In this work the status of the project and some parallel activities on muon radiography are presented.
\end{abstract}

\section{Introduction}

The muon radiography technique, based on the measurement of the absorption degree of cosmic ray muons while traversing a material volume under investigation, was exploited for the first time in 1955 to determine the overburden of rock above a mountain tunnel [1]. Later, between the end of the Sixties and the beginning of the Seventies, there was the first application in the field of Archaeology [2], led by the Nobel Prize particle physicist Louis Alvarez. The experiment concerned the study of the Chefren pyramid in Egypt. Muon radiography allowed Alvarez rejecting the hypothesis of the existence of a yet undiscovered secret room above the main pharaoh's chamber. Following the Alvarez experience recently muon absorption radiography was again taken into consideration, at first by Japanese groups [3] and later by European, Canadian, Mexican and other groups (e.g. [4-8]), to be applied in the fields of Volcanology, Mining and Archaeology. The Progetto Premiale 2012 "MURAVES" (MUon RAdiography of VESuvius) [9] was proposed by Italian groups of the National Institute of Geophysics and Volcanology (INGV), the National Institute for Nuclear Physics (INFN) and the Universities of Naples "Federico II" and of Florence and financed by the Italian Ministry of Education, Universities and Research. It has its roots in the Mu-Ray project [4, 5], an INFN R\&D

\footnotetext{
ae-mail: Lorenzo.Bonechi@fi.infn.it
} 


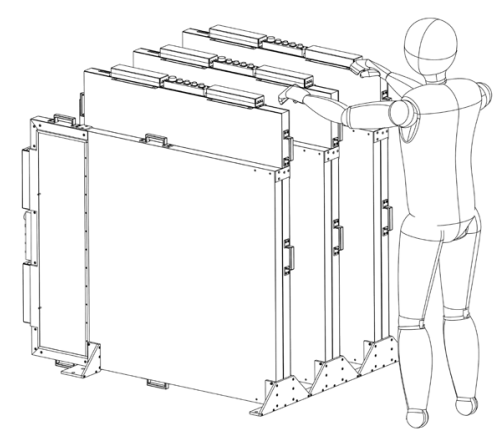

Figure 1. MURAVES base tracking module. In this sketch it is composed by three pairs of tracking planes.

started in 2009 that led to the design of a rugged muon hodoscope based on plastic scintillators and low power electronics, optimized for muon radiography in inhospitable environments. The Mu-Ray hodoscope is the basic system adopted for the MURAVES project, whose main goal is to validate muon radiography as a new tool in the field of Volcanology, by applying this technique to the study of Mount Vesuvius near Naples in Italy, one of the most dangerous active volcanoes in the world due to the sub-plinian behavior of its eruptions together with the massive urbanization of the surrounding areas. According to the new data analysis [10], a large dense body seems to be located just below the Vesuvius crater, down to $2 \mathrm{~km}$ depth. This result is in agreement with an older interpretation based on seismic data. MURAVES will have the possibility to look inside the upper layer of the material located below the base of the crater, thus providing a completely independent measurement of its structure in terms of density.

In section 2 the MURAVES detector, currently in the final stage of the assembling phase, is described. Section 3 gives a short report about two preliminary test measurements on volcanoes carried on thanks to the Mu-Ray prototype detector, while in section 4 the choice of the measurement location for the MURAVES apparatus is shortly discussed. Finally in section 5 some parallel activities, primarily conceived for testing the proposed methodology in more simple cases, are presented.

\section{The MURAVES detector}

The MURAVES apparatus is a modular, robust and low power consumption muon hodoscope optimized to be used in inhospitable environments like the surroundings areas of volcanoes. The complete detection system is a short array of identical and completely independent base tracking modules each of which has an incoming surface of $1 \mathrm{~m}^{2}$. For the study of Vesuvius at least three such base modules will be installed, in such a way to decrease sufficiently the total required exposure time, that will be anyway of several months. In the next paragraphs a description of the apparatus is given with some details.

\subsection{The muon hodoscope}

As already mentioned, the single MURAVES base detector module is a muon tracker derived from the Mu-Ray experience and composed by three or four pairs of detection planes. In figure 1 a sketch of a Mu-Ray-like base tracking module is shown. Each plane provides the necessary information for the reconstruction of one coordinate of a point of the lines traveled by muons crossing the detector. 


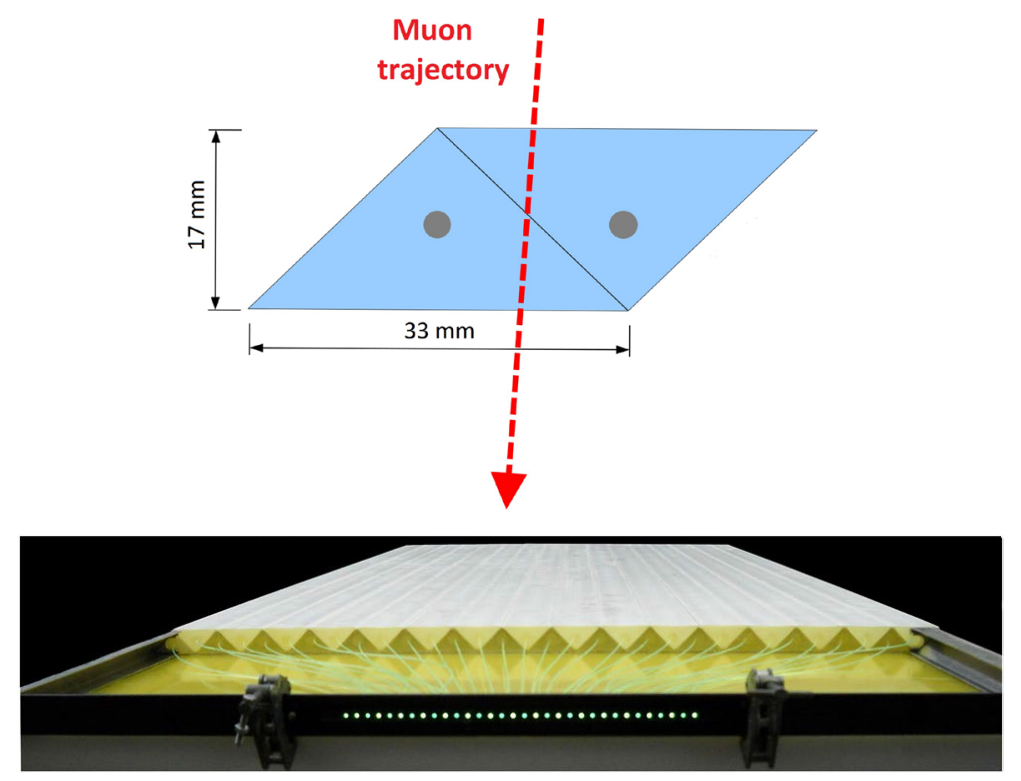

Figure 2. Top: disposition of the scintillator strips allowing the reconstruction of the muon impact point by evaluating the baricenter of the signals produced in adjacent strips. Bottom: a half tracking plane assembled in a dedicated aluminum frame equipped with a plastic holder with 32 through hole to fix the ends of the WLS fibers.

Three pairs of detection planes are sufficient for the univocal identification of muon trajectories, but in the current vision, the possibility of installing four pairs for each tracking module is taken seriously into account for a reason that is explained later in section 4.

The detection plane is an assembly of 64 extruded fast plastic scintillators, in the form of $100 \mathrm{~cm}$ long strips whose section is an isosceles triangle $33 \mathrm{~mm}$ wide and $17 \mathrm{~mm}$ high. The scintillator strips, whose concept was introduced at first for the MINERVA experiment [11], are produced by Fermilab. The basic idea is to build detection planes by disposing these strips side by side alternately arranged with the vertices of the triangles one upwards and the other downwards as shown in the upper side of figure 2. With this particular configuration muons cross usually at least two adjacent bars and it makes possible reconstructing the muon impact points using a baricenter-like algorithm, thus improving the spatial resolution. The scintillator plastic is made of a bulk of polystyrene with the addiction of PPO and POPOP scintillation dopants emitting in the blue wavelength region with an emission maximum at $420 \mathrm{~nm}$. For reading out the scintillator light signal, each extruded scintillator has a $1.5 \pm 0.1 \mathrm{~mm}$ diameter hole, centered along the axis and running for the whole length of the strip. This hole is used to allow the insertion of a $1.2 \mathrm{~mm}$ diameter wavelength shifting (WLS) fiber for the collection of the light signals produced by muons inside the strip. On the lower side of figure 2 a half tracking plane assembled in a mechanical frame and equipped with a plastic holder fixing the ends of 32 WLS fibers is shown. The adopted fibers are $1.2 \mathrm{~mm}$ diameter multiclad Kuraray Y11 S-35 type, characterized by an absorption spectrum approximately in the wavelength range $400 \div 470 \mathrm{~nm}$ and an emission spectrum in the range $470 \div 550 \mathrm{~nm}$, with a peak in the green. An optical sensor optimized to be adapted for the coupling with these WLS fibers has been selected among the models available on the market. It is described in section 2.2. 

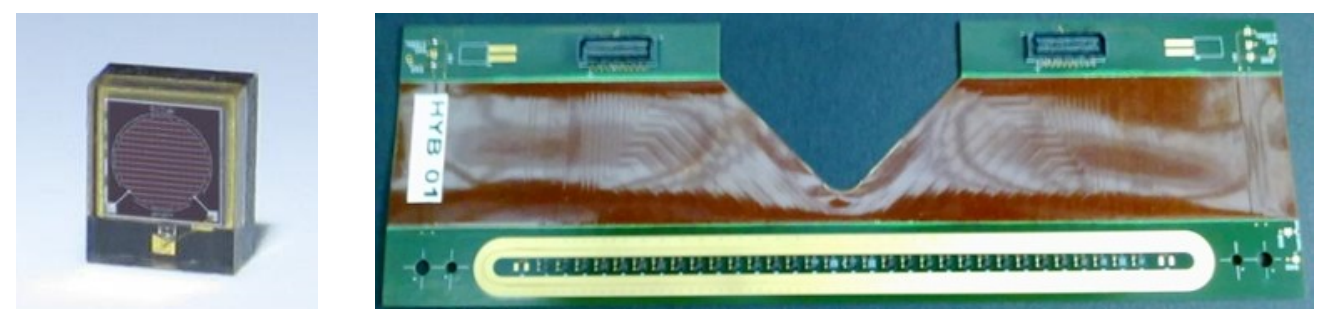

Figure 3. Left: the Advansid ASD-RGB1C-P circular silicon photomultiplier. Right: the MURAVES flexible printed circuit housing $32 \mathrm{SiPM}$.

\subsection{The optical sensor and the readout system}

Many models of silicon photomultipliers have been considered for the use within the Mu-Ray and MURAVES projects, since the beginning on the Mu-Ray R\&D project in 2009. Clearly the quality of the SiPM device has been highly improved by producers since then and recently we have finally compared two models of the last generation: S13360-1350PE by Hamamatsu Photonics [12] and ASD-RGB1C-P by Advansid [13]. Some relevant parameters of both of these models are reported in table 1. The performance are no so dramatically different. The Advansid model has been also characterized in laboratory, measuring at the room temperature the dark rate and the response to real muon signals thanks to a test scintillator system provided with a Kuraray WLS fiber. The model by

Table 1. Comparison of relevant characteristics in standard conditions of operation of two possible optical sensors for the MURAVES detector: Hamamatsu Photonics S13360-1350PE and Advansid model ASD-RGB1C-P.

\begin{tabular}{lcc}
\hline & S13360-1350PE & ASD-RGB1C-P \\
\hline Dark Count Rate $(\mathrm{DCR})\left(\mathrm{kHz} / \mathrm{mm}^{2}\right)$ & 53 & $<100$ \\
PDE $(\%)$ & 40 & 32.5 \\
Operating Voltage (V) & 56 & 30 \\
Gain & $1.7 \times 10^{6}$ & $2.6 \times 10^{6}$ \\
Breakdown Voltage Temperature Coefficient $\left(\mathrm{mV} /{ }^{\circ} \mathrm{C}\right)$ & 54 & 27 \\
\hline
\end{tabular}

Hamamatsu has in general slightly better performance with respect to the model by Advansid. The latter has a higher level of the Dark Count Rate, that is not anyway a dramatic problem, because the detector planes are used in time coincidence, thus allowing rejecting the noise signals randomly generated by the sensors. The Photon Detection Efficiency of the ASD-RGB1C-P model is also lower with respect to the S13360-1350PE model, while the operating voltage is much lower and the stability with temperature is better. Considering that the performance of the ASD-RGB1C-P model by Advansid are suitable for the MURAVES detector and that this model is much cheaper than S13360-1350PE, the ASD-RGB1C-P model has been definitively selected to be finally adopted for the MURAVES detector. In figure 3 the Advansid SiPM optical sensor and the flexible printed circuit designed to house $32 \mathrm{SiPM}$ are presented. The circuit with the SiPM devices is used to collect the photon signals coming from the WLS fibers of a half tracking plane, convert them into electric pulses and send them to a readout board housing a dedicated ASIC, the OMEGA EASIROC chip (Extended Analogue SI-pm ReadOut Chip [14]), which is a 32 channels analogue front-end ASIC dedicated to the readout of silicon photomultipliers. Each of the half planes is provided of such a readout board, 

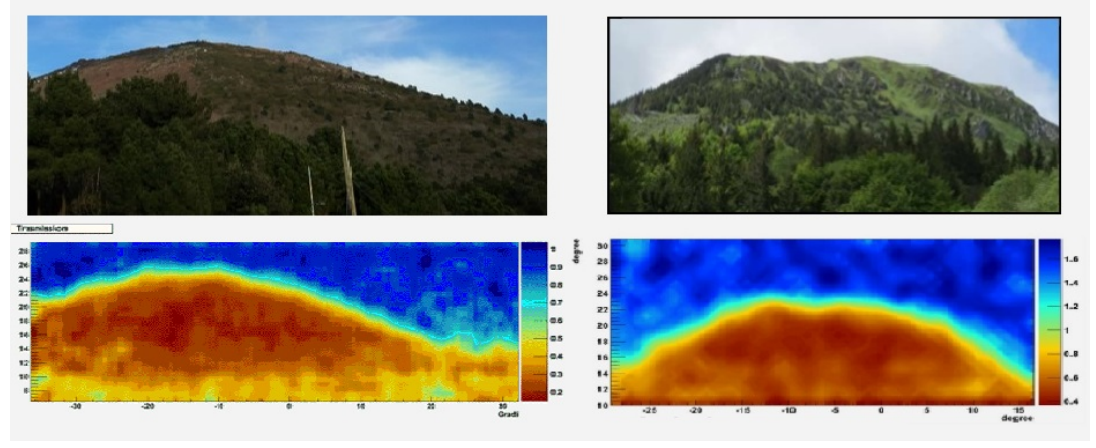

Figure 4. Left: transmission map measured by the Mu-Ray prototype detector at Vesuvius, compared with a picture of Vesuvius taken from the same place. Right: Same as on left, but measured at the Puy de Dome volcano in France.

called also "slave" board, and all the slave boards are finally read out by a "master" board driven by a small Raspberry-PI computer. The master board implements the logic for generating the trigger signal and the sequence of commands for reading out the data from the slave boards, that are finally transferred to the Raspberry-PI and written to a SD memory card.

\subsection{The thermal stabilization system}

The operation of the MURAVES system on the flank of Vesuvius requires a long data taking period, of the order of many months. In this period the detectors will be exposed to sensible variations of temperature due to the different weather conditions and also to the day-night excursion. Due to the variable environment conditions in which the detectors will be requested to operate, a temperature control system has been designed by the INFN Florence Electronics's Pool for the stabilization of the operating temperature of the silicon photomultipliers. These devices are in fact quite sensible to temperature variations and their breakdown voltage has a dependence on the temperature that is quantified by a Breakdown Voltage Temperature Coefficient (BDTC) that for the selected SiPM is $\mathrm{BDTC}=27 \mathrm{mV} /{ }^{\circ} \mathrm{C}$ (see table 1 ). A change in temperature would imply therefore a change in the operating voltage and therefore in the gain of the SiPM sensors. The thermal stabilization system consists of a custom mechanical support between the SiPM detectors and the cooling / heating unit that maximizes the thermal conductivity towards the stabilization element (Thermoelectric Cooler with Peltier effect, TEC) but minimizes the accidental thermal exchange towards the environment. A dedicated control electronics connected to the TEC element manages keeping the temperature of the SiPM sensors constant and uniform and can be programmed via the CAN-Bus interface. In order to minimize the power consumption according to the environmental conditions, the system also controls a fan that has the purpose of optimizing the functioning of the TEC element. The system checks also that the humidity and stabilization temperature conditions do not give rise to condensation near the detector itself.

\section{Test measurements at the Vesuvius and Puy de Dome volcanoes}

The Mu-Ray prototype detector was used between 2014 and 2015 to test the installation and data taking procedures in two different real cases: Mount Vesuvius near Naples (Italy) and Puy de Dome 


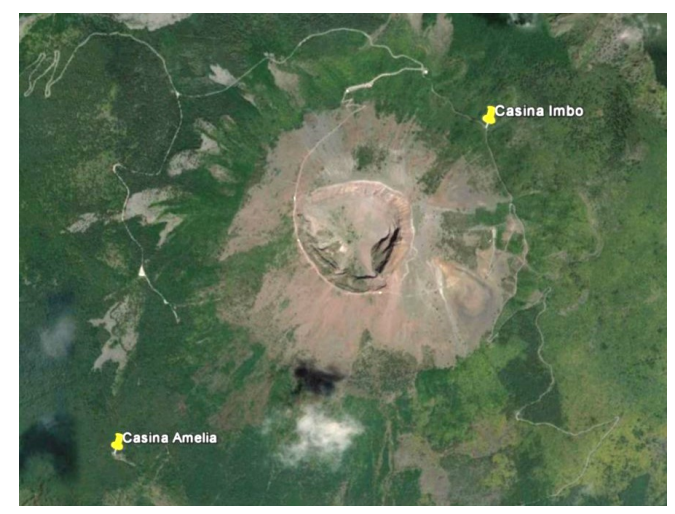

Figure 5. Mount Vesuvius seen on Google Earth; the two locations "Casina Imbo" and "Casina Amelia" have been marked on the map.

near Clermont-Ferrand (France). The latter test was done in collaboration with the TOMUVOL collaboration, a French group working on muon radiography applied to volcanoes. The results of the tests are published in $[15,16]$. These experiments gave us the possibility of testing and improving the data analysis algorithms, allowing finally to produce muon transmission maps and to find the necessary graphic optimization to enhance the visibility of the shadow of the volcanoes. In figure 4 the 2D angular distributions of the measured transmission are shown for both cases. A global agreement of the measured muon fluxes with the expected shadows produced by the volcanoes is found. The collected statistics, corresponding to one week data taking at Vesuvius and few weeks at Puy de Dome, are not enough anyway to distinguish any internal structure of the volcanoes. Furthermore an anomalous muon flux is found at very low elevation angle in the measurement at Vesuvius. The fraction of transmitted muons seems to rise up at low elevation, while it should decrease because of the wider thickness of soil and rock to be traversed. This effect has different contributions. In part it maybe due to the change in the muon energy spectrum at very high zenith angle (horizontal flux). At these angles the total muon flux decrease because the low energy muon component is suppressed. The fraction of very high energy muons is thus higher that for lower zenith angles and therefore the muon radiation is more penetrating. Besides this possible motivation for the observed effect we expect an important contribution by background events due to the scattering of low energy muons on the surrounding soil and rocks. This will be discussed shortly in section 4 .

\section{Identification and selection of the installation area on Mount Vesuvius}

The choice of the location for the installation of the MURAVES apparatus is not simple as it could appear. First of all, the surrounding area of the volcano is classified as a natural park and is managed by the Park Authority "Ente Parco Nazionale del Vesuvio", that is responsible for safeguarding the park and for the authorization of activities within its borders. Furthermore, the area is not perfectly hospitable for the installation of a complex device like the MURAVES detector and the electric power line is not available everywhere. In this scenario, few possible locations have been evaluated so far. The two most promising locations are called "Casina Imbo" and "Casina Amelia". This locations are indicated in figure 5, taken from the Google Earth software. Casina Imbo was selected at first, because it would be the easiest solution from the point of view of the logistic and power line availability. 


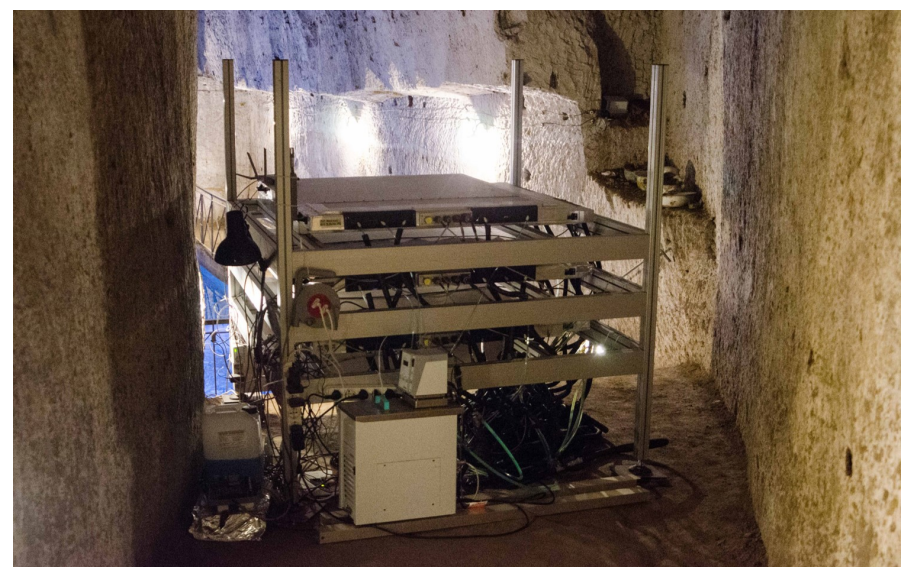

Figure 6. The Mu-Ray prototype detector installed inside the Galleria Borbonica in Naples.

Anyway after recent simulations done by researchers of the French TOMUVOL collaboration using a custom software (the PUMAS Reverse Muon Simulation [17]), who will join the MURAVES collaboration for a joint measurement at Vesuvius with an independent detector, the background events at Casina Imbo could make the measurement vain. In particular, being this location very close to Mount Vesuvius, we expect a not negligible fraction of low energy muons suffering multiple scattering on the mount's outer layers and deflected inside the acceptance of the hodoscope. As we can see from the figure, Casina Amelia is far more distant from the volcano and we expect a lower fraction of such background events. To improve the performance in the reconstruction of the Vesuvius density map the MURAVES and TOMUVOL tracking detectors will be composed of four pairs of tracking planes for each module and a thick Lead layer will be placed downstream the first two pairs. The muon tracks will be reconstructed separately using the upstream pairs and the downstream ones, in such a way that the total multiple scattering angles due to Coulomb interactions of muons inside the Lead layer can be measured event by event. In this way it is possible to identify events affected by large scattering angles, corresponding to the lowest energy muon events, and select only those with energy beyond a threshold of approximately $(1 \div 2) \mathrm{GeV}$. To do this, several tons of Lead blocks will be used. For this reason works are currently in progress to prepare the Casina Amelia site to receive the MURAVES and TOMUVOL detectors, that will be placed inside large metal containers laid down on a strong concrete basement currently under construction.

\section{Other applications under investigation}

Since few years the community working in the field of muon radiography is investigating the possibility of applying these methodologies for geophysical prospections in fields other than Volcanology (see for example the important result reported in [18]). Since 2012 the MURAVES collaboration has done some efforts to understand the performance of muon radiography in the field of Archaeology [19]. The most straightforward task of muon radiography in this field is the search for empty volumes hidden inside larger volumes of massive material. The collaboration has now adopted Archaeology as a primary field of investigation of muon radiography, next to the volcanological one.

In this scenario a first important measurement has been carried out at the Galleria Borbonica archaeological site, a very complex system of underground tunnels and cavities excavated inside 


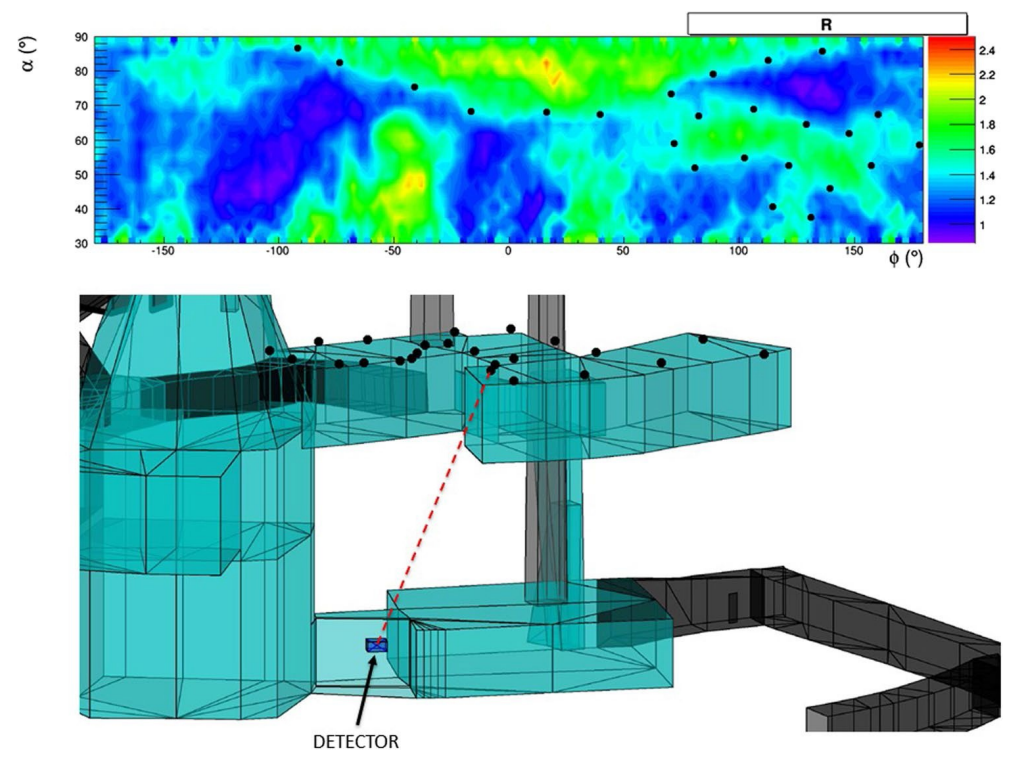

Figure 7. 2D histogram showing the ratio between the measured and the expected muon transmissions at Mount Echia.

Mount Echia, a tuff hill just on the Naples seaside, that originally hosted the ancient Greek colony Parthenope, founded in the $8^{\text {th }}$ century BC. Details are published in [20]. For this measurement, the $1 \mathrm{~m}^{2}$ prototype Mu-Ray detector, composed of three pairs of tracking layers, was placed inside Galleria Borbonica and kept for approximately one month taking data with the detector's axis aligned along the vertical direction. The thickness of the tuff layer above the detector was approximately fifty meters. In figure 6 the Mu-Ray detector in its data taking location is shown. A total of approximately 14.6 million triggers were collected during a data taking time of 26 days. A few centimeters thick Iron/Lead absorber layer was placed between the second and the third tracking layers to define a $100 \mathrm{MeV}$ cutoff for the muon energy. Only very clean events firing all the tracking planes and with a single straight muon track were selected for the final step of the analysis. A 2D histogram of the angular distribution of the reconstructed tracks was produced and compared with a similar histogram produced with a 12 million triggers reference data sample collected outside the gallery, in the INFN laboratories in Naples. After correcting for the slightly different detector's trigger efficiencies and for the different data taking time of the two measurements, the ratio of the two histograms was computed. The result represents the muon flux transmission measured in the detector's field of view inside Galleria Borbonica. The measured transmission was then compared with an expected transmission angular map obtained using a 3D geometrical model of Mount Echia with an average value of the tuff density of $1.71 \mathrm{~g} / \mathrm{cm}^{3}$, the information on the position of the Mu-Ray detector, the muon differential fluxes measured at free sky by the ADAMO project [21] and a parameterization of the muon range in matter (see [20] for details and references). The ratio between the measured and expected transmissions is shown in figure 7. In the lower part of the same figure the system of tunnels located in the Mu-Ray installation area in the Galleria Borbonica is also shown. A set of black points identifying the large tunnel above the detector are highlighted both in the drawing and in the histogram. We observe a general agreement between the location of these points and the 
angular regions where anomalous values of the transmission appear, showing that this system is able to identify the angular location of the known galleries and cavities. In the histogram few zones with anomalous values of transmission are then visible, which are not associated to any know gallery or room, thus pointing to possible unknown cavities.

\section{Conclusions}

The "MURAVES" project is designed for testing the muon radiography technique in the field of Volcanology. The detector, a state of the art rugged and low power muon hodoscope based on plastic scintillators read out by SiPM optical sensors of the last generation, is currently in the final assembling phase. The installation location inside the Mount Vesuvius National Park has been defined and the preparation of the necessary infrastructure is in progress. The location will be prepared to receive both the $3 \mathrm{~m}^{2}$ MURAVES detector and an independent $1 \mathrm{~m}^{2}$ detector by the French TOMUVOL collaboration, for a joint measurement. In parallel, the MURAVES group is carrying on measurement campaigns using a prototype detector in the fields of Archaeology and Geology. Further cases are under consideration in both of these two fields.

\section{Acknowledgments}

The MURAVES collaboration would like to thank the Director of the Galleria Borbonica site, Mr. Gianluca Minin, President of "Associazione Culturale Borbonica Sotterranea", for having hosted us inside Galleria Borbonica, allowing us to test our prospecting methodology in this extremely interesting and beautiful site. We would like also to extend special thanks to Dr. Cristina Carloganu and all the French colleagues of the TOMUVOL group, with whom the MURAVES collaboration has undertaken an interesting collaboration that will take us hopefully to other advantageous joint measurements.

\section{References}

[1] E.P. George et al., Commonw. Eng. 1955, 455-457 (1955)

[2] L.W.Alvarez et al., Science 167, 832-839 (1970)

[3] H.Tanaka et al., Nucl. Inst. and Meth. A507, 657-669 (2003)

[4] A.Anastasio et al., Nucl. Inst. and Meth. A718, 134-137 (2012)

[5] A.Anastasio et al., Nucl. Inst. and Meth. A732, 423-426 (2013)

[6] C.Carloganu et al., Proceedings of Science (EPS-HEP2011) 055 (2011)

[7] http://www.crmgtm.com/

[8] S.Aguilar et al., Proceedings of Science (X LASNPA) 012 (2013)

[9] G.Saracino et al., Annals of Geophysics 60, 1 (2017) S0103

[10] N.Linde et al., Scientific Reports 7, 8434 (2017)

[11] L.Aliava et al., Nucl. Inst. and Meth. A743, 130 (2014)

[12] http://www.hamamatsu.com/

[13] http://www.advansid.com/

[14] S.Callier et al., Phys. Procedia 37, 1569-1576 (2012)

[15] F.Ambrosino et al., JINST 9 C02029 (2014)

[16] F.Ambrosino et al., J. Geophys. Res. Solid Earth, 120

[17] V.Niess et al., arXiv:1705.05636 [physics.comp-ph] 
[18] K.Morishima et al., Nature 552, 386-390 (2017)

[19] L.Viliani, Master Degree in Physics, University of Florence (Italy), 2011/2012; title: La radiografia muonica di strutture sotterranee con un odoscopio: studio della fattibilità e primi sviluppi (in Italian)

[20] G.Saracino et al., NATURE Scientific Reports 7, 1181 (2017)

[21] L.Bonechi et al., Proceedings of the $29^{\text {th }}$ International Cosmic Ray Conference (Pune, India) Vol. 9, 283-286 (2005) 